\title{
Review
}

\section{A Review of Color Flavor Interaction in Food and its Application in Food Product Development}

\author{
Shane T. McDonald, PhD* \\ Principal Flavor Chemist, Kalsec ${ }^{\circledR}$ Inc., 37 I 3 West Main Street, Kalamazoo 49006, Michigan, USA \\ *Corresponding author \\ Shane T. McDonald, PhD \\ Principal Flavor Chemist, Kalsec ${ }^{\circledR}$ Inc., 37I 3 West Main Street, Kalamazoo 49006, Michigan, USA; E-mail: SMcDonald@kalsec.com
}

\section{Article information}

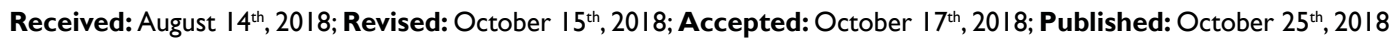

\section{Cite this article}

McDonald ST. A review of color flavor interaction in food and its application in food product development. Adv Food Technol Nutr Sci Open J. 20 I8; 4(I): 23-27. doi: I0.17|40/AFTNSOJ-4-149

\begin{abstract}
Humans use all of the senses, especially sight and ortho-nasal olfaction, to create an expectation of what a flavor of a food will be before ingestion. The expectation is confirmed or disconfirmed when the food is placed in the mouth by taste (gustation) and retronasal olfaction. Color is an important constituent of sight. Colors that create the correct expectation of a flavor are called "congruent" and colors that create incorrect expectations are termed "discongruent". Studies have shown that the ability of people to correctly identify flavor is increased considerably if the color is congruent, or expected, with the flavor. Congruently colored foods have been shown to increase the perception of sweetness in beverages, the richness of orange juice, the pungency of salsa, and the richness of chocolate. Incongruent colored foods may create an incorrect perception of the foods flavor, which many consumers find surprising and unpleasant. For that reason, it is usually best to develop foods that are congruently colored. However, there are opportunities to create excitement and other positive benefits from deliberately coloring foods in a discongruent fashion. More research is needed on this topic, especially in the areas of the effect of minor differences of shades of colors in food flavor perception, and how to successfully implement a strategy of discongruence in food product design.
\end{abstract}

Keywords

Natural colorant; Color flavor interaction; Beverages; Fruit product design.

\section{INTRODUCTION}

"You eat with your eyes"? What does that mean? Humans make their first judgements on food based on appearance and confirm with taste and smell. One of the most important visual characteristics of appearance is color.

Flavor is the sensory impression of food and beverage when taken into the mouth. It is mostly determined by the senses of taste and smell. However, all of the senses are involved in the perception of food. As an illustration, you see a hamburger. At the same time, flavor compounds volatilize off the hamburger and escape into the air. The chemicals are swept up into the nose and are detected by receptors in the olfactory nerve, a process called "orthonasal olfaction", that is, from the external environment into the nose. This information is used to create a perception in the brain: it expects the food to taste like a hamburger. You then bring the food into your mouth and chew. Non-volatile tastants are perceived by the taste buds as sweet, sour, bitter, salty, and umami. Other tactual senses are noted such as temperature, texture, sound, and chemesthetic effects. Flavor compounds volatilize off the food mass and are swept through the back of the throat into the nose and again detected by the olfactory bulb: this is called "retronasal olfaction", from the inside of the mouth into the nose. The sensory information from the mouth and retronasal olfaction confirm or disconfirm the expectation.

As will be discussed, humans are very poor at identifying flavors without visual information. And a very important constituent of visual information is the color of the food.

\section{LITERATURE REVIEW}

Food color is well-known to affect the consumer's perception of the flavor of the food. ${ }^{1}$ It affects the aesthetics, safety, sensory characteristics, and acceptability of food. Color is a major con- 
tributor for initial acceptance/rejection of food. Early in cognitive development, one learns to associate foods with specific colors.

There have been numerous studies on how the color of food affects the perception of flavor. In a famous study by Dubose et al, ${ }^{2}$ panelists were asked to taste and identify coloredmasked samples of retail, fruit-flavored, non-carbonated beverages. Panelists did a poor job of identifying the flavors by tasting without seeing the color of the beverage. Seventy percent of panelists correctly identified grape, but only $20 \%$ identified orange. However, when allowed to see the colors of the beverages, identification was almost $100 \%$.

When the color of the beverage was typical for the flavor (red for cherry, green for lime, orange for orange, and colorless for flavorless), flavor identification was greatest. However, when the color was inappropriate (or incongruent, which means unexpected), flavors were often incorrectly perceived. For example, $33 \%$ of panelists thought a red-colored lime-flavored beverage was a red fruit (cherry, strawberry, raspberry). Fourty percent thought a green-colored, flavorless beverage was lemon-lime flavor.

Other studies suggested that color can affect the perceived intensity of sweetness, ${ }^{3}$ the intensity of pungency perception in salsa ${ }^{3,4}$ the acceptability of fruit juice, ${ }^{1}$ and the intensity of chocolate flavor. ${ }^{4}$ All pretty amazing considering that the color doesn't change the flavor or wholesomeness of the product. The color of a product gives an indication of the odor, flavor and texture of the food. Through pattern recognition, a consumer associates certain food colors with certain food flavors, so visual clues would influence food choice and acceptability.

Early on, it became apparent in investigating color/flavor interaction that the story was complicated. Many (over 100) studies have been done on this phenomenon. ${ }^{5}$ It has been noted that many of these studies have been contradictory. So many, it appears that the simple results of experiments may be only part of the story.

In everyday life and some professional activities (sensory analysis, perfumery and flavor creation, oenology) humans make use of their abilities to identify odors. ${ }^{6}$ The odors of wine tend to be grounded by the colors of the wine. In oenological tasting, a single taster can provide a wide variety of comments. Comments are based on analytical descriptions of the visual, olfactory and gustatory properties of the wine. The analysis of wine tasting is well adapted for studying interactions between various sensory modalities. Determination is modified if the color of the wine is obscured by use of an opaque glass. Acceptance of wine is significantly correlated to its color.

Morot $^{6}$ study four extensive bodies of tasting wine tasting notes (corpuses). For the most part, red wine descriptors are represented by red or dark objects, while the white wine with yellow or clear objects, which was common for all four corpuses. Red wines had descriptors such as prune, cocoa, raspberry, and tobacco. White wine descriptors included "melon", "citrus", "butter" and "apple". It was determined separately that it is difficult to determine if a wine is red or white when tasted in opaque glasses: $70 \%$ got it right, which is better than chance but is shows that it is difficult to determine a red wine $v s$. white wine without visual assistance.

The panelists were given two wines. One was a white wine " $W$ ' and one was a white wine dyed red " $R W$ '. It was determined separately that the two wines were identical in taste and that the dye did not affect the flavor. Panelists tasted the two sets of wine and overwhelmingly used white wine descriptors to describe $\mathrm{W}$ and red wine descriptors to describe RW.

The white wine was perceived to have the odor of red wine when colored red. The wine's color provides significant sensory information, which misleads the subject's ability to judge flavor. The mistake is stronger in the presence that in the absence of access to the wine color.

The subjects smell the wine, make the conscious act of odor determination and verbalize their olfactory perception by using odor descriptors. However, the sensory and cognitive processes were mostly based on wine colors.

The study concluded that the sense of smell is, by itself, unlikely to provide sufficient information to allow for a consciously reasoned decision. The capacity to identify odors could only be an accessory aspect of the olfactory function.

A similar study was performed on beer. ${ }^{7}$ They differentiated "experts" (people with technical knowledge of beer as well as in-depth sensory exposure), "trained assessors" (sensory panelists with several years training in beer attributes, but generally without the technical knowledge of beer) and "novices" (beer drinkers but without sensory or technical training). This study involved trained assessors and novices; either sighted or blindfolded evaluation of three styles of beers (blond, amber, and dark) from each of three breweries. It was assumed that when sighted, the trained assessors would discriminate based on brewery and not beer color, unlike the novices who would go by color first.

The results were that, when blindfolded, both groups tended to categorize beers by brewery, detecting the different characteristic qualities of each brewer. When sighted, both groups categorized by color. So, when blindfolded, both groups could categorize by brewery but when sighted they used color? This was even though the trained assessors claimed that they ignored color when they were evaluating the beer. The researchers had two hypotheses on what happened. One, when blindfolded, the panelists concentrated on chemosensory properties such as bitterness, mouthfeel, and aroma. When sighted, they couldn't process both visual and chemosensory information at the same time, so they used visual information and ignored smell and taste information. This is called selective attention. The other theory was that the assessors were able to switch attention from color to chemosensory attributes, but the chemosensory information was driven by visual information. That is, when analyzing the beers, 
they were seeking to confirm the visual information rather than analyzing taste and smell information. This is called "congruence seeking". This may be why the trained assessors felt they relied more on chemosensory information when in fact they relied on visual information.

Cultural expectations also come to play. ${ }^{5}$ What happens when a Taiwanese and a Briton taste a brown liquid which is in fact a grape product? This question was studied using Taiwanese and British panels. The Taiwanese expected grape flavored product and a tart taste. Upon tasting the beverage, their expectation will be confirmed, and the brown color enhanced the flavor of grape. The Briton tasted the beverage expecting the sweet taste of cola. Instead, it was tart, and they received a "disconfirmation" of expectation. They could either "assimilate", that is, perceive the drink to be sweeter than it is to match expectation, or "contrast", the color expectation has no effect on sweetness perception. Assimilation can occur if there is not a great discrepancy between expected and actual, or the stimulus is ambiguous, allowing for the panelist to focus on qualities congruent with expectations, or simply social pressure/experimental demands to align perception with group expectation. Otherwise, contrast will occur.

Zellner, ${ }^{8}$ reviewed of color-odor interactions. She contrasted sensations that perceived food "out there" (color and orthonasal olfaction which detects aroma) and food in your mouth (gustation, retronasal olfaction, trigeminal stimulation). Because of the co-occurrence of visual and olfactory inputs, there should be no big surprise that the two senses interact.

People tend to perceive intense colored food as having stronger odors. Some color-odor combinations are quite common, such as brown and caramel and strawberry and pink/red. However, as mentioned by Shankar ${ }^{5}$ with their studies of color/ flavor perception from different cultures, much color-flavor association is learned.

Color exerts its influence on odor identification, discrimination, intensity and pleasantness through its ability to activate an odor image and indirectly through its ability to facilitate retrieval of odors labelled from memory, which can also active an odor image. So, many odors have been acquired and are stored in memory. As mentioned above, humans tend not to have unique odor words (labels) like taste (sweet, sour, bitter). Rather, the words used are objects that possess the aroma. Think banana, fruity, grilled, smoky, etc. So, with no visual clue, when presented with an aroma without a context, such as a colorless liquid in a jar, upon smelling the odor you discover a match through a "library" of retained odor objects. This takes a long time.

However, with a color clue, the mind activates olfactory "images" of all odors corresponding to the color and creates a precept that is a fusion of all these images. The odors associated with that color will form a larger portion of the precept. For instance, cherry is a flavor associated with the color red. The color-induced precept will contain more cherry odor than odors not associated with the color.
Also, color will help retrieve an odor label which will help in odor identification. It helps narrow the possible odor labels to those that have been associated with that color in the past. It also fuses the color and label precepts with the actual flavor.

These labels will have the ability to transform the perception of the actual odor into ones that most closely resembles the odor image activated by the label. The color activates a list of odor labels that the mind associates with that color. An appropriate color will increase the likelihood that the correct odor image will be retrieved, and the correct odor image will be chosen as a match for the actual odor. After the label is chosen and an odor image is activated from memory, the label transforms the actual odor perception into one more closely resembling the memory, giving rise to a very strong, clear odor experience. Thus, olfactory intensity, identification, and enjoyment of the odor will all be increased by the presence of an appropriate color.

What if an inappropriate color is used? The wrong odor images will be recalled, which are inconsistent to the aroma smelled. The subject may be unable to find an appropriate label. This will result in the perception of a strong, unpleasant aroma. Alternatively, the aroma can be mis-identified, and the perceived odor judged as unpleasant example of the flavor, or the mind can transform to one more congruent with the color.

Violations of expectations can affect retronasal olfaction. The color causes the aroma (orthonasal) perception to be enhanced. If the perception of the color-enhanced aroma is not met, the retronasal perception can be weaker in a colored solution than in a clear solution. Inappropriate-colored odors may be perceived as unpleasant in the mouth due to violated expectation.

\section{COLOR FLAVOR INTERACTION IN FOOD PRODUCT DEVELOPMENT /}

In summary, sight and orthonasal olfaction create an expectation of flavor. Taste and retronasal olfaction confirm or disconfirm the expectation. Humans find it difficult to identify odors without visual clues. When the color helps created the correct flavor expectation, the color and flavor are "congruent". When they do not, they are "discongruent".

When the color and flavor are congruent, a number of advantageous effects occur, including mentioned above and summarized in Table 1.

\begin{tabular}{cc}
\hline \multicolumn{2}{|c|}{ Table I. Positive Effects of Congruent Color Flavor Interaction in Foods } \\
\hline Increased flavor recognition & Dubose $^{2}$ \\
\hline Increase perception of richness in orange juice & Clydesdale $^{1}$ \\
\hline Increase perception of pungency in salsa & Shermer and Levitan $^{4}$ \\
\hline Increase perception of sweetness in beverages & Roth $^{3}$ \\
\hline Increased chocolate flavor perception & Shermer and Levitan $^{4}$ \\
\hline Assist in recognition of beer style & Lelievre $^{7}$ \\
\hline
\end{tabular}

In most cases, the product developer would want the 
color to create a perception that is accurate and favorable to the flavor of the product. Bear in mind that this color and flavor combination must survive processing and remain congruent through the shelf life of the product.

Color flavor incongruency can lead to a number of negative results, as summarized by Table 2 . As descried by Dubose ${ }^{2}$ above, incongruecy leads to poor flavor identification.

The risk is surprising a customer, who may then dislike the product as the flavor did not meet the expectation. An example of this was a "dinnerparty" where the attendees ate steak, peas and French fries under color-masking conditions. When half-way through the meal, the lighting was changed to allow the true color of the food, it was revealed that the steak was blue, the peas were red, and the French fries were green. Many of the attendees were physically ill at the unexpected color change. ${ }^{9}$

Another example of discongruency is a cola soft drink (typically brown in color from the use of caramel coloring) that was formulated to be clear and colorless. Many consumers were confused by the cola taste of a beverage that looked like a lemonlime soft drink..$^{10,11}$ As described earlier by Shankar, ${ }^{5}$ a color may suggest a particular flavor to one ethnic group and be an example of positive congruency but be incongruent for another with consumer dislike and confusion. Morot ${ }^{6}$ described how panelists would incorrectly described white wine when colored red.

Dubose $^{2}$ and much of the literature cited above describes very obvious color flavor incongruency (e.g. green and cherry). However, work by Garber ${ }^{12}$ indicate that even small differences in the colors used to represent flavors can significantly affect the consumer's ability to correctly identify the flavor as well as the perceived flavor profile and preferences.

\begin{tabular}{|cc}
\hline \multicolumn{2}{|c|}{ Table 2. Negative Effects of Color Flavor Discongruency in Foods } \\
\hline $\begin{array}{c}\text { Poor flavor identification } \\
\text { Product dislike as color and flavor do not match }\end{array}$ & Dubose $^{2}$ and others \\
\hline $\begin{array}{c}\text { Confusion on product identity and taste perception } \\
\text { in wine and soft drinks }\end{array}$ & Morot $^{6}$ and Murray \\
\hline $\begin{array}{c}\text { Misidentification of flavor due to cultural or ethnic } \\
\text { associations }\end{array}$ & Shankar $^{5}$ \\
\hline
\end{tabular}

However, opportunities exist for deliberately mismatching color and flavor. Because consumers have strong color/flavor associations, deliberately mismatching them can be quite attention getting. ${ }^{10}$

He details three ways to successfully implement this strategy. Once was to teach the consumer a new association. Examples include the brown in colas (from caramel color) and green in mint (mint oil is colorless). A second way is to celebrate the new color, such as certain soft drinks which are colored a vivid green. A third technique is to break the color/flavor association completely so color is not suggestive of the flavor. This can easily be accomplished by opaque packaging. Some companies also use names that are not suggestive of flavor at all (think of "sunset breeze" or "winter chill").

Examples of positive results in deliberate discongruency of color and flavor in foods is summarized in Table 3. Glass ${ }^{13}$ described the efforts of a major food manufacturer to create new colors of catsup. Typically, almost always red in color, this company sought to excite children with green catsup, and later explored other colors. One concern with using novel coloring is that color is that, if successful, color can be easily duplicated by competitors. Also, consumers may initially be excited by the new color, then quickly lose interest and return to the traditional coloring.

Raspberries are usually red, as are strawberries, cherries, and watermelons. To reserve a shade of red just for raspberries, food manufactures used the color amaranth (FD\&C Red \#2) for raspberry flavored products. When the Food \& Drug Administration (FDA) banned the use of Red\#2, manufacturers switched to the cheap but relatively unused FD\&C Blue \#1. The justification for the color change was that this was the flavor of "Blue Raspberries" (Rubus leucodermis), despite the fact that neither the color nor the flavor was really accurately represented by the blue raspberry flavor. Blue raspberry flavor, with its brilliant blue color, is now common in many confections and frozen desserts. ${ }^{14}$

The perception of different flavors can be accomplished by using the same flavor, but in different colored products. ${ }^{15}$ One cereal manufacturer has a product with cereal pieces of different colors, including red, yellow and orange, which are traditionally associated with flavors such as cherry or strawberry, lemon and orange. In fact, the cereal pieces all have the same flavor.

Vegetarian "burgers" often try to simulate a beef hamburger in flavor and appearance. At least two companies have gone further by coloring the interior of the burger red to make it look like the bleeding of a rare to medium hamburger. ${ }^{16,17}$ The manufactures are not trying to pass their burgers as real meat. They feel that the plant protein composition is a competitive advantage for various reasons. However, the red coloring enhances the perception of the beef flavor in the burger.

\begin{tabular}{|c|c|}
\hline Excitement from untraditional color in catsup & Glass $^{13}$ \\
\hline New flavor categories (such a blue raspberry) & Conservapedia $^{14}$ \\
\hline Perception of multiple flavors in cereal & Adams $^{15}$ \\
\hline $\begin{array}{l}\text { Intensify beef flavor perception in meat-free } \\
\text { products }\end{array}$ & Robertson $^{16}$. Camlee ${ }^{17}$ \\
\hline
\end{tabular}

\section{CONCLUSIONS}

Many studies have demonstrated that color of a food will impact the perception of the flavor. Consumers will often let the color override odor in the perception of the flavor of the product. Coloring of food, when it creates the correct perception of the flavor of the food ("congruency"), will increase the intensity, character, and intensity of the flavor. When the color creates an incorrect perception of the flavor ("discongruency"), the consumer may be 
confused and dislike the product. In certain applications discongruency can be beneficial.

Much of the research that has been done in this field has resulted in conflicting results. There is still a lot of research needed to determine the mechanisms and effects of color/flavor interaction. In most cases, food color should be congruent with the flavor. However, much of the research has been done using with obvious discongruency. It is generally obvious that a lemonflavored food should not be colored red. More work needs to be done on minor differences of shades. What shades of red should cherries, strawberries, and watermelon flavored foods be? How can color be used to distinguish a fresh, wild, ripe, or cooked strawberry flavor? The food product developers can use this information to better design their products.

Another area of interest is how to use discongruent color and flavor interaction. In most cases, this can result in customer dissatisfaction, but there are examples of this concept being used quite successfully. Research on this subject will decrease the risk of this method and result in more successful, exciting and innovative product launches.

\section{REFERENCES}

1. Clydesdale F. Color as a factor in food choice. Crit Rev Food Sci Nutr. 1993; 33(1): 83-101. doi: 10.1080/10408399309527614

2. DuBose C, Cardello A, Maller O. Effects of colorants and flavorants on identification of perceived flavor intensity, and hedonic quality of fruit-flavored beverages and cake. J Food Sci. 1980; 5(5): 1393-1415. doi: 10.1111/j.1365-2621.1980.tb06562.x

3. Roth H, Radle L, Gifford G, Clydesdales F. Psychophysical relationships between perceived sweetness and color in lemonand lime-flavored drinks. J Food Sci. 1988; 53(4): 1116-1119. doi: 10.1111/j.1365-2621.1988.tb13543.x

4. Shermer D, Levitan C. Red hot: The cross modal effect of color intensity on perceived piquancy. Multisens Res. 2014; 27(3-4): 207223.

5. Shankar M, Levitan C, Spence C. Grape expectations: The role of cognitive influences in color-flavor interactions. Conscious Cogn. 2010;19(1): 380-390. doi: 10.1016/j.concog.2009.08.008

6. Morot G, Brochet F, Dubourdieu D. The color of odors. Brain Lang. 2001; 79(2): 309-320. doi: 10.1006/brln.2001.2493
7. Lelievre M, Chollet S, Herve A, Valentin D. Beer-trained and untrained assessors rely more on vision than on taste when they categorize beers. Chem Percept. 2009. doi: 10.1007/s12078-009-9050-8

8. Zellner D. Color-odor interactions: A review. Chem Perception. 2013 ; 6: 155-169.

9. Garber L, Hyatt E, Starr R. The effects of food color on perceived flavor. J Marketing Theory and Practice. 2000; 8(4): 59-72. doi: 10.1080/10696679.2000.11501880

10. Garber L, Hyatt E, Nafees L. The effects of color on perceived flavor: A factorial investigation in India. Journal of Food Products Marketing. 2016; 22(8): 930-948. doi: 10.1080/10454446.2014.885864

11. Murray K. Lessons from Crystal Pepsi. Website: http://theconversation.com/enabling-innovation-lessons-from-crystal-pepsi-84967. Accessed October 13, 2018.

12. Garber L, Hyatt E, Nafees L. The effects of analogous food color on perceived flavor: A factorial investigation. I Food Products Marketing. 2016; 22(4): 1-5. doi: 10.1080/10454446.2015.1072866

13. Glass S. What were they thinking? The day ketchup crossed the line from perfect to purple. The fast company. Website: https:// www.fastcompany.com/1779591/what-were-they-thinking-dayketchup-crossed-line-perfect-purple. Accessed October 13, 2018.

14. Conservapedia.com. Blue raspberry flavor. Website: https:// www.conservapedia.com/Blue_raspberry_flavor. Accessed October 13, 2018.

15. Adams C. Are different colors of froot loops different flavors? The strait dope. Website: http://www.straightdope.com/columns/ $\mathrm{read} / 1683 /$ are-the-different-colors-of-froot-loops-different-flavors/. Accessed October 13, 2018.

16. Chamlee V. Why do people want veggie burgers that bleed? Eater.com. Website: https://www.eater.com/2016/7/25/12270698/ lab-grown-meat-beyond-burger-impossible-foods. Accessed October 13, 2018.

17. Robertson M. The bill gates-backed veggie burger that "bleeds" is going into the grocery store meat cases. Business Insider. Website: https://www.businessinsider.com/beyond-meat-veggie-burger-safeway-2017-5. Accessed October 13, 2018. 